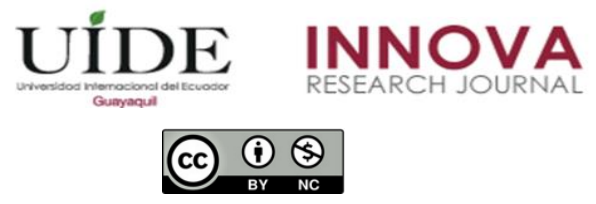

INNOVA Research Journal, ISSN 2477-9024

(Septiembre-Diciembre 2020). Vol. 5, No.3.1 pp. 58-75

DOI: https://doi.org/10.33890/innova.v5.n3.1.2020.1579

URL: http://revistas.uide.edu.ec/index.php/innova/index

Correo: innova@uide.edu.ec

\title{
Liderazgo directivo y su impacto en el aprendizaje estudiantil en instituciones educativas
}

\section{Executive leadership and its impact on student learning in educational institutions}

Judith Soledad Yangali Vicente

(Dhttp://orcid.org/0000-0003-0302-5839

Gian Carlos Torres Lévano

(1) https://orcid.org/0000-0002-9721-6638

Universidad Norbert Wiener, Perú

Autor para correspondencia: judithsyv@gmail.com; gian.carlos.torres.levano@ gmail.com

Fecha de recepción: 01 de octubre de 2020 - Fecha de aceptación: 06 de noviembre de 2020

\begin{abstract}
Resumen
El objetivo general de la investigación fue determinar la correlación entre la percepción del liderazgo directivo y el aprendizaje estudiantil en instituciones educativas del Callao. La metodología utilizada fue el enfoque cuantitativo, prospectivo, de corte transversal, de alcance correlacional y diseño no experimental. La muestra estudiada fue en base a una población censal de 20 docentes entre 20 a 60 años y a una población censal de 20 alumnos pertenecientes al $6^{\circ}$ grado de primaria. Los datos fueron recolectados mediante una encuesta basada en un cuestionario sobre estilos de liderazgo y un registro de notas estandarizado. Se realizó el análisis estadístico mediante el software SPSS versión 22.0. Los datos fueron registrados en términos de medidas descriptivas. Los resultados arrojaron que existe un grado de correlación bajo entre las variables (Rho de Spearman: 0,205) con nivel bilateral de significancia de 0.05 , es decir a un $95 \%$ de confianza. Por lo tanto, se concluye que no existe impacto directo ni significativo entre la percepción del liderazgo directivo y el aprendizaje estudiantil en las instituciones educativas del Callao.
\end{abstract}

Palabras claves: aprendizaje estudiantil; calidad educativa; gestión educativa; liderazgo directivo.

\begin{abstract}
The general objective of the research was to determine the correlation between the perception of directive leadership and student learning in educational institutions of Callao. The methodology used was the quantitative, prospective, cross-sectional, correlational scope and non-experimental design approach. The sample studied was based on a census population of 20 teachers between 20 and 60 years old and a census population of 20 students belonging to 6th grade of primary school. Data were collected through a survey based on a questionnaire on leadership styles and a standardized grade register. Statistical analysis was performed using SPSS version 22.0 software. The data were recorded in terms of descriptive measures. The results showed that there is a low
\end{abstract}


degree of correlation between the variables (Spearman's Rho: 0.205) with a bilateral level of significance of 0.05 , that is, at $95 \%$ confidence. Therefore, it is concluded that there is no direct or significant impact between the perception of directive leadership and student learning in the educational institutions of Callao.

Keywords: student learning; educational quality; educational management; directive leadership.

\section{Introducción}

La educación a finales del siglo XX y principios del XXI, se ha visto influenciada por muchos factores internos y externos que demandan cambios relevantes a nivel mundial. Durante el presente siglo, han surgido una serie de políticas educativas desfavorables para mejorar la educación peruana. La mayoría de reformas o leyes ofrecidas desde el Estado han consolidado las brechas existentes entre los grupos de poder y los pobres; éstos últimos han experimentado la exclusión y manipulación desde el ámbito educativo. Ciertamente, es una necesidad actual solucionar con éxito los requerimientos de la exigente y cambiante sociedad en la que vivimos, en plena era de avances científicos y tecnológicos.

El primer factor preponderante en la calidad de gestión educativa lo constituye el liderazgo del director, seguido por el desempeño docente; ambos evidenciados como producto final en el aprendizaje estudiantil. Desde esta perspectiva, se puede afirmar que un proceso educativo de calidad se caracteriza por las más altas capacidades de los profesionales involucrados en su desarrollo. Según la Organización de las Naciones Unidas para la Educación, la Ciencia y la Cultura (UNESCO, 2016), el liderazgo directivo implica conducir a las instituciones educativas hacia niveles superiores de aprendizaje, creando condiciones que favorezcan el desarrollo profesional y desempeño de los docentes.

Por lo tanto, es importante resaltar los sucesos recientes ocurridos en el Perú dentro del ámbito educativo; en el año 2003, surge la reformada Ley General de Educación № 28044. Por otro lado, el año 2006 aparece la Ley del Sistema Nacional de Evaluación, Acreditación y Certificación de la Calidad Educativa (SINEACE) N 28740. En el 2007, el Ministerio de Educación (MINEDU) establece el Proyecto Educativo Nacional hacia el 2021 y se suscribe la nueva Ley de Carrera Pública Magisterial (CPM) N 29062. Posteriormente, en el 2008, se promueve el Programa Nacional de Formación y Capacitación Permanente (PRONAFCAP), que ha propiciado la mejora del desempeño docente, dando énfasis a la formación en las áreas de razonamiento lógico-matemático y comprensión lectora manifiesta Reyes (2012).

En el año 2014, el Congreso de la República aprueba la "Nueva Ley Universitaria” en medio de una polémica, la cual estableció la creación de la Superintendencia Nacional de Educación Universitaria (SUNEDU), institución que reemplaza a la Asamblea Nacional de Rectores (ANR) y que está encargada de supervisar la calidad universitaria, fiscalizar el uso de recursos y autorizar la apertura de nuevas universidades.

Sin embargo, el desarrollo de la gestión educativa traducida en mejora de la "calidad educativa continua" a nivel nacional es una asignatura aún pendiente por resolver. Se observan directivos sin la capacidad adecuada para solucionar problemáticas institucionales y de gestión pedagógica, del mismo modo otros ni siquiera presentan disposición al cambio para potenciar el 
ámbito educativo. Por lo consiguiente, esta investigación pretende analizar el liderazgo directivo relacionado directamente al aprendizaje estudiantil en instituciones educativas primarias de la Provincia Constitucional del Callao.

\section{Figura 1}

Organigrama de Variables de Investigación.

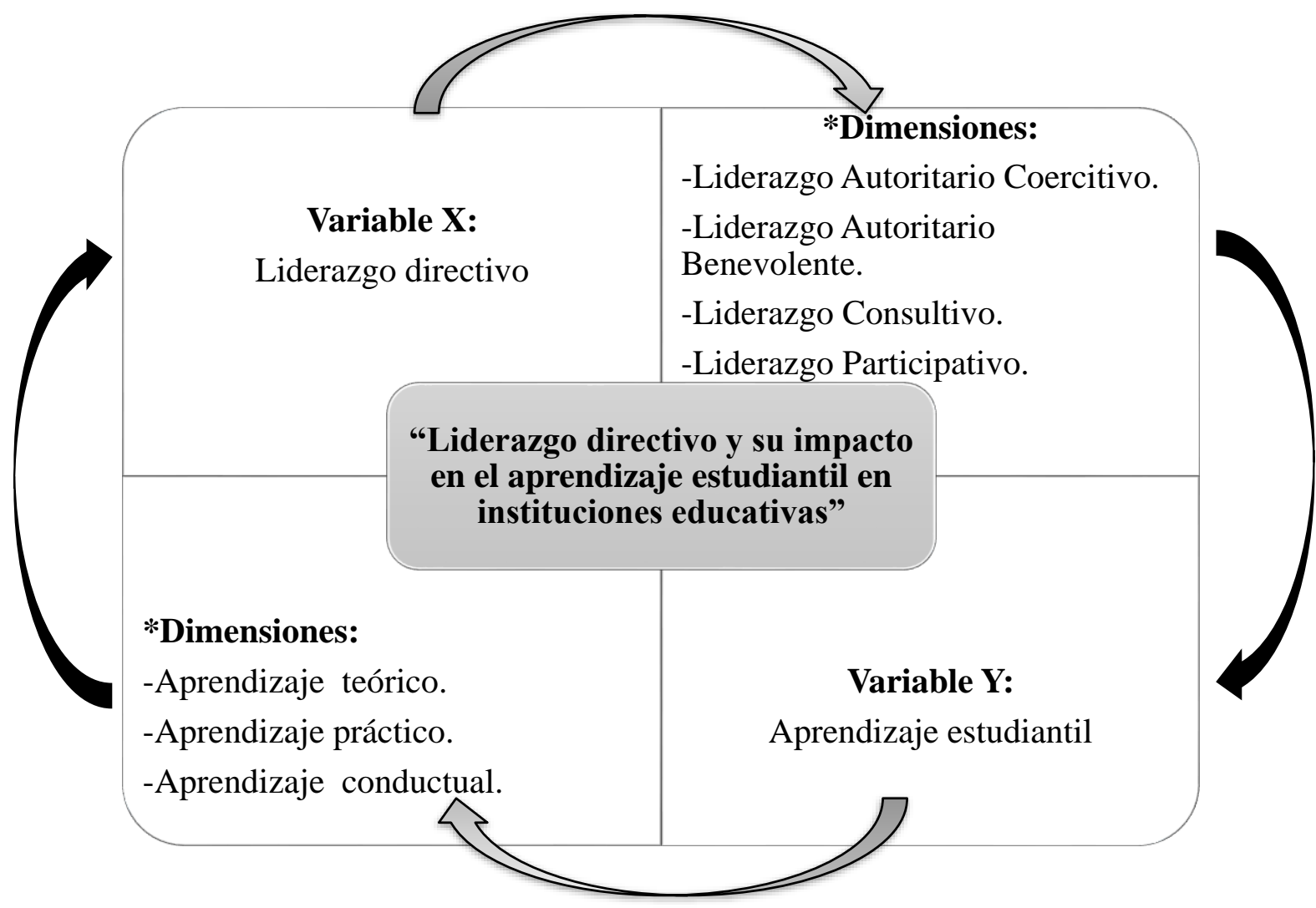

\section{Marco Teórico}

\section{Liderazgo directivo}

Se inicia con definir al liderazgo directivo como "un fenómeno social, por ello se concibe como la influencia interpersonal ejercida en determinada situación para la concreción de uno o más objetivos". Estos planteamientos sirven de plataforma para afirmar que el liderazgo abarca un proceso continuo impulsado por una visión basada en la influencia, el poder personal, credibilidad para cambiar y aceptar a los docentes y a su ambiente manifiesta Chiavenato (2004). Por lo tanto, los directivos deben establecer una dirección para desarrollar una visión de futuro donde el aprendizaje de diferentes estrategias permita producir los cambios necesarios. Por ello, el líder apoya, ayuda y transmite seguridad aclara Furguerle y Vitorá (2016). 
Todo director educativo debe reunir una serie de características personales y habilidades de gestión, con las cuales pueda lograr con éxito las metas con la participación activa de los principales actores educativos, véase docentes y estudiantes. En este mismo sentido, se afirma lo siguiente: "definir los valores que se han de promover no resulta sencillo porque se tiene perspectivas diferentes más aun, los propios valores matizan la respuesta"; es decir, el directivo clarifica los valores a promover dentro de la institución refiere Garza y Patiño (2000).

Por su parte Hernández, Santo y González (2012), afirman que entre las características, capacidades y habilidades de un líder sobresalen las siguientes:

a) Físicas, como la capacidad de trabajo y resistencia a la fatiga.

b) Intelectuales, que incluyen la inteligencia media, alta y la fluidez verbal.

c) Sociales, constituidas por la comunicación, motivación, sociabilidad, sensibilidad social o empatía, simpatía, trabajo en equipo, ecuanimidad, asertividad, cooperación, confiabilidad, delegación y apoyo.

d) Psicológicas, como el equilibrio, control, autoconfianza, estabilidad emocional, tenacidad, resistencia a la frustración, iniciativa y creatividad.

e) Prácticas, conformadas por el conocimiento, información, aptitudes específicas, eficacia y la responsabilidad.

Es relevante precisar las diferencias entre liderazgo y dirección, a menudo esto dos términos se emplean como sinónimos; sin embargo, es necesario diferenciar el significado de cada uno de ellos. Según Palomo (2010) los líderes y directivos son dos tipos de personas diferentes, los objetivos de los directivos surgen, a diferencia de los líderes, de la necesidad más que del deseo, así como tampoco adoptan actitudes personales y activas respecto a los objetivos, mantienen relaciones de trabajo poco estrechas por lo que su entorno laboral es controlado. Líderes y directivos se diferencian esencialmente en su forma de orientación hacia los objetivos, la concepción del trabajo, sus relaciones interpersonales y sus características de personalidad. A continuación, se detallan las principales diferencias:

\section{Tabla 1}

Diferencias entre liderazgo y dirección.

\begin{tabular}{ll}
\hline \multicolumn{1}{c}{ Liderazgo } & \multicolumn{1}{c}{ Dirección } \\
\hline $\begin{array}{l}\text { Comprende un propósito común que } \\
\text { favorece lograr cambios reales en la } \\
\text { organización; genera crecimiento y } \\
\text { bienestar. }\end{array}$ & $\begin{array}{l}\text { Abarca la coordinación de los trabajadores y } \\
\text { los recursos para desarrollar las actividades de } \\
\text { la organización. }\end{array}$ \\
$\begin{array}{l}\text { Elabora una visión en la organización. } \\
\text { Origina cambio y construye nuevas } \\
\text { realidades. }\end{array}$ & $\begin{array}{l}\text { Organización en el presente. } \\
\text { Respalda el funcionamiento en una realidad } \\
\text { específica. }\end{array}$ \\
\hline
\end{tabular}




\section{Liderazgo}

Impacta en los trabajadores.

Las diferentes actividades que desempeñan

los líderes buscan la eficacia.

Influye sobre recursos emocionales, valores, compromisos y principios.

La relación es de motivación e influencia.

Desde cualquier posición ejerce.

Convierte los problemas en oportunidades de solución.

\section{Dirección}

Engloba el producir, cumplir y asumir responsabilidades.

Las actividades buscan la eficiencia.

La acción afecta los recursos: materias primas, habilidades y tecnología.

La relación es de autoridad.

Requiere una posición desde la que ejerce.

Toma decisiones para resolver problemas.

Fuente: elaboración propia, basada en Campodonico (2017) y Palomo (2010).

Durante muchas décadas el estilo habitual de muchos directivos fue el estilo autoritario, basado en el poder que ejercía sobre el grupo de trabajo; en la actualidad, se espera de un líder un estilo más participativo en el que la responsabilidad y la autoridad reside en un equipo de trabajo o equipo directivo. La investigación indica que está emergiendo un tipo de liderazgo que se denomina liderazgo distribuido o liderazgo compartido, en el que básicamente un principal líder influye en sus colaboradores dándoles poder y autonomía para actuar y tomar decisiones aclara Campodonico (2017). Tras revisar la abundante bibliografía se resaltó la teoría sobre el liderazgo de Likert porque se relaciona mejor con el presente estudio y también con las dimensiones del instrumento de recolección de datos a emplear.

Fue Likert (1968), el cual estableció la presencia de 4 dimensiones basadas en estilos y características de la dirección dentro de una empresa, véase: autoritarismo-coercitivo, autoritarismo-benevolente, consultivo y participativo. Estos epígrafes fueron por primera vez incluidos en su obra New Patterns of Management de 1961. Después fueron modificados respectivamente a sistema I, II, III, y IV. A continuación, se precisan las dimensiones del liderazgo directivo según Likert:

El liderazgo autoritario coercitivo, se basa radicalmente en la cima gerencial, en consecuencia, es monopolizada toda decisión unidireccional por la gerencia que siempre se direcciona de forma vertical descendente. También, evidencia una comunicación descendente de característica pobre para formular acciones a cumplir, resaltándose una notoria distancia entre el director y sus trabajadores segregados de los cargos gerenciales. Se precisa la realización condicionada por miedo a los castigos y sanciones incluidas en el reglamento interno de la institución. Además, se crea una informal empresa nociva. 
Liderazgo autoritario benevolente: Se presenta con los subordinados cierta confianza condescendiente y tolerante traducida en órdenes que se les formula dentro de un marco de control interno. La informal empresa que surge de forma inesperada se considera aún como una amenaza posible para la evolución de la empresa. Asimismo, los correctivos y/o castigos se manifiestan con disminuida arbitrariedad y las recompensas sociales aparecen ocasionalmente.

Liderazgo consultivo, favorece la consulta a los trabajadores y la delegación de funciones, sin embargo, la decisión final siempre la posee el director. El cual tiene parcial confianza en sus trabajadores; suele considerar constructivamente las opiniones e ideas de los mismos, sin embargo, las pertinentes decisiones derivan de la gerencia. La comunicación es de tipo discrecional, se orienta en el sentido descendente y horizontal. Aquí los trabajadores poseen parcial autonomía en su trabajo fomentándose su actuación dentro de procesos de control.

Liderazgo participativo, se caracteriza porque los directivos poseen confianza total en el trabajo de sus subordinados, en la toma de decisiones es el más pertinente y rentable.

Considerándolo se otorgan reconocimientos sociales y recompensas económicas, los castigos son muy extraños y se deciden de forma colectiva, se difunde el proceso de metodologías innovadoras, su comunicación es asertiva y bidireccional, entre directivo y subordinado hay proximidad psicológica, la toma de decisiones es colaborativa, etc.

\section{Aprendizaje estudiantil}

Se define al aprendizaje estudiantil como la adquisición por la práctica de una conducta duradera según la Palomino (2012). Esta definición pertenece a la estructura de educación, por tanto, la educación constituye el sistema de aprendizaje. La acción y efecto de aprender algún arte, oficio u otra cosa es compleja. Asimismo, afirma que "el aprendizaje es un proceso y propone un ciclo de aprendizaje que se basa en 3 fases: exploratoria, introductoria y de aplicación".

Existe un factor determinante a la hora que un individuo aprende y es el hecho de que hay algunos alumnos que aprenden ciertos temas con más facilidad que otros, para entender esto, se debe trasladar el análisis del mecanismo de aprendizaje a los factores que influyen, los cuales se pueden dividir en dos grupos : los que dependen del sujeto que aprende (la inteligencia, la motivación, la participación activa, la edad y las experiencia previas) y los inherentes a las modalidades de presentación de los estímulos, es decir, se tienen modalidades favorables para el aprendizaje cuando la respuesta al estímulo va seguida de un premio o castigo, o cuando el individuo tiene conocimiento del resultado de su actividad y se siente guiado y controlado por una mano experta manifiesta Palomino (2012).

Son cuatro las teorías de aprendizaje que mayoritariamente se usan en la actualidad: las inteligencias múltiples, el conductismo, el cognitivismo y el constructivismo; las cuales se proceden a describir:

a) Inteligencias múltiples; el psicólogo Gardner (1983), plantea que "la gente nace con ciertas aptitudes o inteligencias para aprender nueva información y resolver problemas". Según este autor, la mayoría sólo se siente cómoda con tres o cuatro de 
estas. La siguiente tabla enumera y describe estas nueve inteligencias y las preferencias de los estudiantes para cada una de ellas.

Tabla 2

Las nueve inteligencias múltiples de Howard Gardner

\begin{tabular}{|c|c|c|}
\hline Inteligencia & Descripción & Preferencias de los Estudiantes \\
\hline Inteligencia Lingüística & $\begin{array}{l}\text { Aptitud para } \\
\text { escribir y hablar }\end{array}$ & $\begin{array}{l}\text { Los estudiantes prefieren aprender a través de } \\
\text { comunicación escrita y verbal. }\end{array}$ \\
\hline $\begin{array}{l}\text { Inteligencia } \\
\text { Lógica/Matemática }\end{array}$ & $\begin{array}{l}\text { Aptitudes para } \\
\text { matemáticas y } \\
\text { lógica }\end{array}$ & $\begin{array}{l}\text { Los estudiantes prefieren aprender a través de } \\
\text { análisis lógico, estrategias y creación de } \\
\text { procesos. }\end{array}$ \\
\hline $\begin{array}{l}\text { Inteligencia Rítmica } \\
\text { Musical }\end{array}$ & $\begin{array}{l}\text { Aptitud para el } \\
\text { movimiento físico }\end{array}$ & $\begin{array}{l}\text { Los estudiantes prefieren aprender a través de } \\
\text { movimiento y tacto, al igual que a través de } \\
\text { experiencia física. }\end{array}$ \\
\hline $\begin{array}{l}\text { Inteligencia } \\
\text { Corporal/Kinestésica }\end{array}$ & $\begin{array}{l}\text { Aptitud para el } \\
\text { movimiento físico }\end{array}$ & $\begin{array}{l}\text { Los estudiantes prefieren aprender a través de } \\
\text { movimiento y tacto, al igual que a través de } \\
\text { experiencia física. }\end{array}$ \\
\hline $\begin{array}{l}\text { Inteligencia } \\
\text { Espacial/Visual }\end{array}$ & $\begin{array}{l}\text { Aptitud para } \\
\text { visualizar cosas }\end{array}$ & $\begin{array}{l}\text { Los estudiantes prefieren aprender a través de } \\
\text { imágenes, formas y diseños visuales. }\end{array}$ \\
\hline Inteligencia Naturalista & $\begin{array}{l}\text { Aptitud para estar } \\
\text { en la naturaleza }\end{array}$ & $\begin{array}{l}\text { Los estudiantes prefieren aprender a través } \\
\text { del trabajo con elementos vivos al igual que } \\
\text { con las diferentes características del mundo } \\
\text { natural. Esta aptitud es especialmente valiosa } \\
\text { entre los biólogos, botánicos y granjeros. }\end{array}$ \\
\hline $\begin{array}{l}\text { Inteligencia } \\
\text { Intrapersonal }\end{array}$ & $\begin{array}{l}\text { Aptitud para } \\
\text { trabajar en } \\
\text { solitario }\end{array}$ & $\begin{array}{l}\text { Los estudiantes con esta aptitud aprenden } \\
\text { mejor a través del auto descubrimiento. }\end{array}$ \\
\hline $\begin{array}{l}\text { Inteligencia } \\
\text { Interpersonal }\end{array}$ & $\begin{array}{l}\text { Aptitud para } \\
\text { trabajar con otras } \\
\text { personas }\end{array}$ & $\begin{array}{l}\text { Los estudiantes prefieren aprender a través } \\
\text { del trabajo en grupo y la comunicación. }\end{array}$ \\
\hline Inteligencia Existencial & $\begin{array}{l}\text { Aptitud para } \\
\text { entender el } \\
\text { propósito propio }\end{array}$ & $\begin{array}{l}\text { Los estudiantes prefieren aprender a través de } \\
\text { realizar y responder preguntas acerca de las } \\
\text { realidades existenciales como la vida y la } \\
\text { muerte. }\end{array}$ \\
\hline
\end{tabular}

Fuente: elaboración propia, citando a Gardner (1983). 
b) El conductismo; se basa en los cambios observables y cuantificables del comportamiento. Se asume que el comportamiento del alumno se forma mediante refuerzos positivos o negativos. Los refuerzos, tanto positivos como negativos, aumentan la probabilidad de que el comportamiento vuelva a ocurrir. El castigo, por el contrario, disminuye la probabilidad de que el comportamiento vuelva a suceder. Algunos de sus representantes más reconocidos fueron: Pavlov con el acondicionamiento clásico, Thorndike con el conexionismo, Watson con la observación de la conducta humana y Skinner con el condicionamiento operante describe Schunk (2012).

c) El cognitivismo; se considera como un proceso de aprendizaje activo. Según los cognitivistas, el aprendizaje implica la reorganización de las experiencias. El cognitivismo asume que se utiliza una estructura de conocimiento existente para procesar información nueva. Esta teoría considera que la información es recibida, almacenada y recuperada. Cuando los cognitivistas diseñan sus cursos, se centran principalmente en el alumno. Sus representantes más relevantes fueron: Bruner con el aprendizaje por descubrimiento y Bandura con la simbolización de la información cognitiva expresa Schunk (2012).

d) El constructivismo; se centra cómo los estudiantes construyen el conocimiento basándose en la experiencia previa. Ellos creen en el aprendizaje experiencial y auto-dirigido. Entre sus representantes destacan: Piaget con la asimilación acomodación y el equilibrio, Ausbel con el aprendizaje significativo y Vigotsky con el proceso interpersonal del aprendizaje afirman Araya, Alfaro, y Andonegui (2007).

Propone Popham (2008) que la evaluación del aprendizaje estudiantil implica "un intento formal de determinar el estatus de los estudiantes con respecto a las variables educativas de interés". Se sabe que la evaluación es un proceso complejo, pues engloba diferentes aspectos del proceso educativo relacionados al desarrollo y logro de competencias propuestas en un currículo. Es por ello que, radica la importancia de resumir brevemente los métodos de evaluación del aprendizaje en la tabla siguiente:

\section{Tabla 3}

Métodos de evaluación del aprendizaje.

\begin{tabular}{ll}
\hline Categoría & Definición \\
\hline Observaciones directas & Ejemplos de conducta que demuestran aprendizaje. \\
Exámenes escritos & Desempeño por escrito en pruebas, cuestionarios, tareas, \\
& trabajos y proyectos. \\
Exámenes orales & Preguntas, comentarios y respuestas verbales durante la \\
Calificaciones de terceros & enseñanza. \\
\hline
\end{tabular}




\begin{tabular}{|c|c|}
\hline Categoría & Definición \\
\hline $\begin{array}{ll}\text { - } & \text { Autorreportes } \\
\text { - } & \text { Cuestionarios } \\
\text { - Entrevistas } \\
\text { - Recapitulación dirigida } \\
\text { - Pensamiento en voz alta } \\
\text { - Diálogos }\end{array}$ & $\begin{array}{l}\text { Juicios de los observadores sobre los atributos que indican } \\
\text { el aprendizaje de sujetos } \\
\text { Juicios de las personas sobre sí mismas. } \\
\text { Respuestas escritas a reactivos o respuestas a preguntas } \\
\text { Respuestas orales a preguntas. } \\
\text { Recuerdo de los pensamientos que acompañaban a la } \\
\text { ejecución de una tarea en un momento dado. } \\
\text { Verbalización de los propios pensamientos, acciones y } \\
\text { sentimientos mientras se desempeña una tarea } \\
\text { Conversaciones entre dos o más personas. }\end{array}$ \\
\hline
\end{tabular}

Fuente: elaboración propia, citando a Schunk (2012).

Del mismo modo, es relevante precisar las características de la evaluación del aprendizaje:

Integral; debido a que desde la perspectiva del aprendizaje se involucran las diversas dimensiones como: la social, intelectual, motriz, afectiva y valorativa de los estudiantes. En tal sentido, la evaluación formativa considera un enfoque de conocimientos, actitudes y sociocultural evidenciada en el currículo, debido a que su objeto es medir la adquisición de competencias básicas, transversales y específicas según el MINEDU (2009).

Procesal; porque se aplica durante el transcurso del proceso educativo, tomando como referencia sus distintos momentos: al inicio, durante y al final; de manera que los resultados contribuyan a la toma de decisiones pertinentes para potenciar y garantizar el aprendizaje estudiantil inmerso en un marco de mejora continua y responsabilidad social, refiere Palomino (2012).

Sistemática; puesto que da respuesta a los propósitos educacionales previamente establecidos, y basados en ellos se aplica, mediante indicadores o criterios coherentes. Los resultados que emite favorecen la orientación de procesos, rediseñar las programaciones, incluir nuevas estrategias de solución ante cualquier eventualidad conflictiva explica Palomino (2012) citando a Serrano (2002).

Participativa; porque favorece la participación activa de todos los actores educativos en el proceso evaluativo de manera óptima, involucrando principalmente al estudiante, docente, directivo y a los padres de familia en la mejora de la calidad de los aprendizajes, mediante la continua autoevaluación, coevaluación y heteroevaluación fundamenta objetivamente Palomino (2012).

Flexible; debido a que se puede adaptar a las diferencias particulares de cada estudiante, respetando sus propios estilos y ritmos de aprendizaje. En función de estas diferencias se seleccionan y definen las técnicas e instrumentos de evaluación más pertinentes. Es bueno que 
los docentes apliquen instrumentos diferentes para evaluar los aprendizajes, pues así se estará atendiendo a la mayoría de estudiantes explica el Ministerio de Educación del Perú (2009).

\section{Materiales y Métodos}

La investigación se desarrolló con un enfoque cuantitativo, cuyo objetivo fue determinar el impacto que ejerce el liderazgo directivo en el aprendizaje estudiantil; según el tiempo de ocurrencia fue prospectivo, de corte transversal, de alcance correlacional y diseño no experimental. La muestra estudiada fue en base a una población censal de 20 docentes (contratados y nombrados de ambos sexos) entre 20 a 60 años y a una población censal de 20 alumnos (de ambos sexos) pertenecientes al $6^{\circ}$ grado de primaria. Los instrumentos de investigación empleados fueron una encuesta basada en un cuestionario sobre estilos de liderazgo aplicado a los docentes, y un registro de notas estandarizado a nivel nacional por el Ministerio de Educación del Perú (MINEDU) de los alumnos. La encuesta fue previamente validada a través del juicio de 3 expertos. Asimismo, se aplicó la prueba Alfa de Cronbach para establecer su confiabilidad, se obtuvo un índice de 0,805; es decir, una fuerte confiabilidad. Cada dimensión de la encuesta sobre estilos de liderazgo, consta de 4 indicadores (total: 16 indicadores) a ser evaluado mediante una escala de Likert, tal como se muestra en la Tabla 4.

\section{Tabla 4}

Matriz de operacionalización de la variable liderazgo directivo $(X)$.

\begin{tabular}{|c|c|c|c|}
\hline Dimensiones & Indicadores & Ítem & Escala \\
\hline \multirow{6}{*}{$\begin{array}{l}\text { Liderazgo } \\
\text { autoritario } \\
\text { coercitivo }\end{array}$} & AC1. Nivel de permeabilidad en el proceso & 1 & Nunca \\
\hline & decisorio en el estilo autoritario coercitivo. & 2 & Raramente \\
\hline & AC2. Nivel de desarrollo del sistema de & 3 & Ocasionalmente \\
\hline & comunicación en el estilo autoritario coercitivo. & 4 & Usualmente \\
\hline & $\begin{array}{l}\text { AC3. Nivel de evolución de las relaciones } \\
\text { interpersonales en el estilo autoritario coercitivo. }\end{array}$ & 5 & Siempre \\
\hline & $\begin{array}{l}\text { AC4. Nivel de ejecución del sistema de } \\
\text { recompensas y castigos en el estilo autoritario } \\
\text { coercitivo. }\end{array}$ & & \\
\hline \multirow{4}{*}{$\begin{array}{l}\text { Liderazgo } \\
\text { autoritario } \\
\text { benevolente }\end{array}$} & $\begin{array}{l}\text { AB1. Nivel de permeabilidad en el proceso } \\
\text { decisorio en el estilo autoritario benevolente. }\end{array}$ & & \\
\hline & $\begin{array}{l}\text { AB2. Nivel de desarrollo del sistema de } \\
\text { comunicación en el estilo autoritario benevolente. }\end{array}$ & & \\
\hline & $\begin{array}{l}\text { AB3. Nivel de evolución de las relaciones } \\
\text { interpersonales en el estilo autoritario } \\
\text { benevolente. }\end{array}$ & & \\
\hline & $\begin{array}{l}\text { AB4. Nivel de ejecución del sistema de } \\
\text { recompensas y castigos en el estilo autoritario } \\
\text { benevolente. }\end{array}$ & & \\
\hline $\begin{array}{l}\text { Liderazgo } \\
\text { consultivo }\end{array}$ & $\begin{array}{l}\text { C1. Nivel de permeabilidad en el proceso } \\
\text { decisorio en el estilo consultivo. }\end{array}$ & & \\
\hline
\end{tabular}




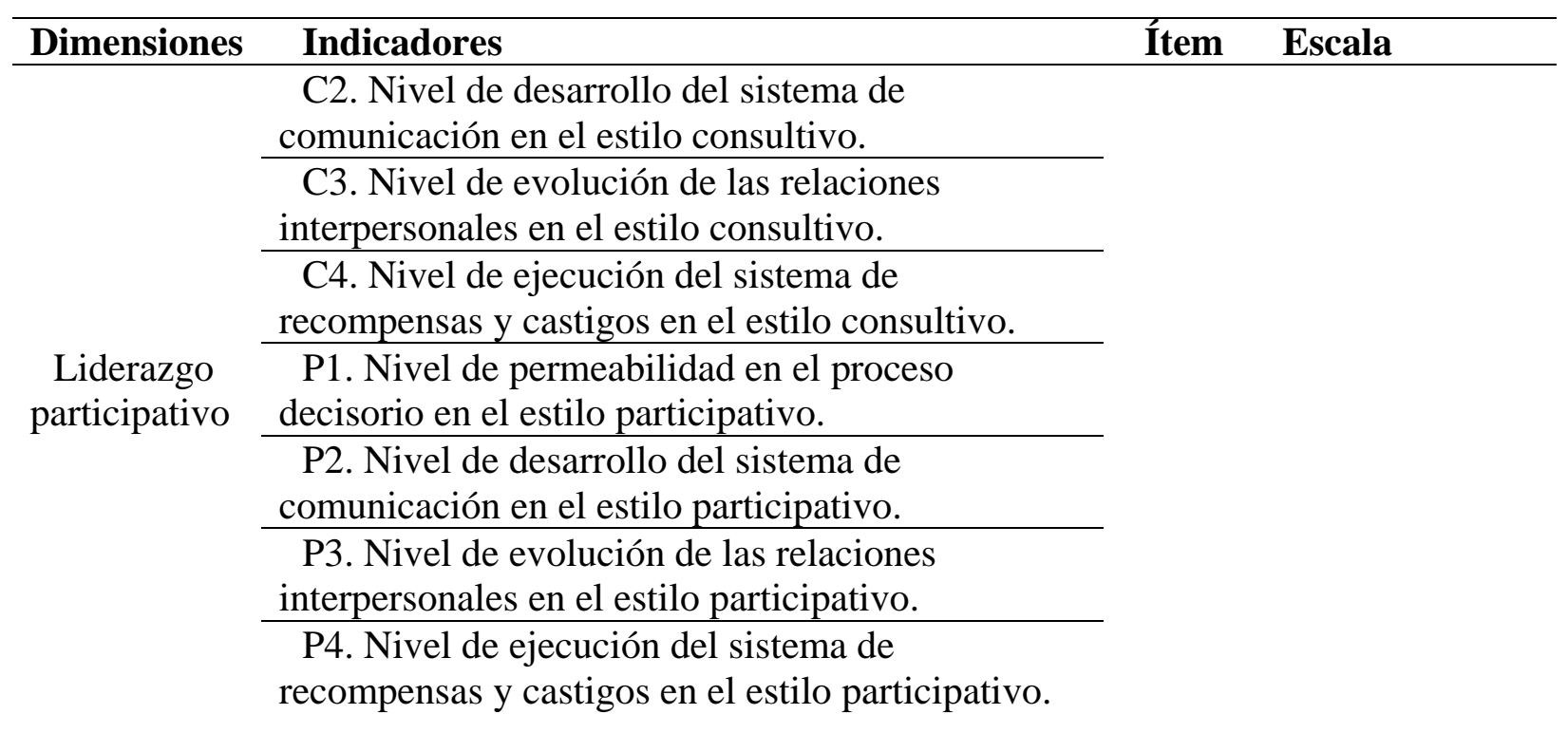

Fuente: elaboración propia.

Al igual que el caso anterior, el registro de notas estandarizado por el MINEDU fue previamente validada a través del juicio de 3 expertos. Asimismo, se aplicó la prueba Alfa de Cronbach para establecer su confiabilidad, se obtuvo un índice de 0,790; es decir, una fuerte confiabilidad. El mismo se aplicó de manera permanente todo el año académico 2019. Las dimensiones analizadas fueron: el aprendizaje teórico, aprendizaje práctico y conductual. Cada dimensión considerada en el registro de notas, consta de 3 indicadores (total: 12 indicadores) a ser evaluados mediante la escala Likert, tal como se muestra en la Tabla 5.

\section{Tabla 5}

Matriz de operacionalización de la variable aprendizaje estudiantil (Y).

\begin{tabular}{|c|c|c|c|}
\hline Dimensiones & Indicadores & Ítem & Escala \\
\hline \multirow{4}{*}{$\begin{array}{l}\text { Aprendizaje } \\
\text { teórico }\end{array}$} & PT1. Promedio teórico del $1^{\circ}$ trimestre. & 1 & AD (logro destacado) \\
\hline & PT2. Promedio teórico del $2^{\circ}$ trimestre. & 2 & A (logro previsto) \\
\hline & PT3. Promedio teórico del $3^{\circ}$ trimestre. & 3 & $\mathrm{~B}$ (en proceso) \\
\hline & PTPF. Promedio teórico ponderado final & 4 & $\mathrm{C}$ (en inicio) \\
\hline \multirow{4}{*}{$\begin{array}{l}\text { Aprendizaje } \\
\text { práctico }\end{array}$} & PP1. Promedio práctico del $1^{\circ}$ trimestre. & & \\
\hline & PP2. Promedio práctico del $2^{\circ}$ trimestre. & & \\
\hline & PP3. Promedio práctico del $3^{\circ}$ trimestre. & & \\
\hline & PPPF. Promedio práctico ponderado final. & & \\
\hline \multirow{4}{*}{$\begin{array}{l}\text { Aprendizaje } \\
\text { conductual }\end{array}$} & PC1. Promedio conductual del $1^{\circ}$ trimestre. & & \\
\hline & PC2. Promedio conductual del $2^{\circ}$ trimestre. & & \\
\hline & PC3. Promedio conductual del $3^{\circ}$ trimestre. & & \\
\hline & $\begin{array}{l}\text { PCPF. Promedio conductual ponderado } \\
\text { final. }\end{array}$ & & \\
\hline
\end{tabular}

Fuente: elaboración propia. 


\section{Resultados}

\section{Resultados Inferenciales: Prueba de Hipótesis}

Hipótesis general

1) Planteamiento de la hipótesis

H0: No existe relación directa y significativa entre la percepción del liderazgo directivo y el aprendizaje estudiantil en las instituciones educativas del Callao, 2019.

H1: No existe relación directa y significativa entre la percepción del liderazgo directivo y el aprendizaje estudiantil en las instituciones educativas del Callao, 2019.

2) Nivel de significancia: $\alpha=5 \%$

3) Criterio de decisión:

Si p-valor $<\alpha$, Se rechaza la hipótesis nula,

Si p-valor $>\alpha$, No se rechaza la hipótesis nula

4) Estadístico de prueba

\section{Tabla 6}

Prueba de correlación para contrastación de hipótesis general.

\begin{tabular}{|c|c|c|c|c|}
\hline & & & Variable X & Variable Y \\
\hline \multirow[t]{6}{*}{$\begin{array}{c}\text { Rho de } \\
\text { Spearman }\end{array}$} & \multirow[t]{3}{*}{$\begin{array}{l}\text { Variable X: } \\
\text { Liderazgo directivo }\end{array}$} & $\begin{array}{l}\text { Coeficiente de } \\
\text { correlación }\end{array}$ & 1,000 & ,205 \\
\hline & & Sig. (bilateral) & . & ,387 \\
\hline & & $\mathrm{N}^{\circ}$ & 20 & 20 \\
\hline & \multirow[t]{3}{*}{$\begin{array}{l}\text { Variable Y: } \\
\text { Aprendizaje estudiantil }\end{array}$} & $\begin{array}{l}\text { Coeficiente de } \\
\text { correlación }\end{array}$ & ,205 & 1,000 \\
\hline & & Sig. (bilateral) & ,387 & 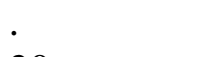 \\
\hline & & $\mathrm{N}^{\circ}$ & 20 & 20 \\
\hline
\end{tabular}

Fuente: elaboración propia.

Los resultados obtenidos evidencian que existe un grado de correlación bajo entre las variables (Rho de Spearman: 0,205) a un nivel bilateral de significancia de 0.05, es decir a un 95\% de confianza. Debido a que el nivel crítico es inferior al nivel de significación establecido existen suficientes razones para rechazar la hipótesis general y se concluye que no existe impacto directo ni significativo entre el liderazgo directivo y el aprendizaje estudiantil. 


\section{Tabla 7}

Prueba de correlación para contrastación de hipótesis específica 1.

\begin{tabular}{|c|c|c|c|c|}
\hline & & & $\begin{array}{c}\text { Variable X } \\
\text { (dimensión 1) }\end{array}$ & Variable $\mathbf{Y}$ \\
\hline \multirow[t]{6}{*}{$\begin{array}{c}\text { Rho de } \\
\text { Spearman }\end{array}$} & Variable $\mathbf{X}$ : dimensión 1 & $\begin{array}{l}\text { Coeficiente de } \\
\text { correlación }\end{array}$ & 1,000 & ,406 \\
\hline & \multirow{5}{*}{$\begin{array}{l}\text { Liderazgo autoritario } \\
\text { coercitivo } \\
\text { Variable Y: } \\
\text { Aprendizaje estudiantil }\end{array}$} & Sig. (bilateral) & $\cdot$ & ,076 \\
\hline & & $\mathrm{N}^{\circ}$ & 20 & 20 \\
\hline & & $\begin{array}{l}\text { Coeficiente de } \\
\text { correlación }\end{array}$ & ,406 & 1,000 \\
\hline & & Sig. (bilateral) & ,076 & $\cdot$ \\
\hline & & $\mathrm{N}^{\circ}$ & 20 & 20 \\
\hline
\end{tabular}

Fuente: elaboración propia.

En la Tabla 7 se observa que existe un grado de correlación moderado entre la dimensión 1 de la variable X y la variable Y (Rho de Spearman: 0.406) a un nivel bilateral de significancia de 0.05 , es decir a un $95 \%$ de confianza. Debido a que el nivel crítico es inferior al nivel de significación establecido existen suficientes razones para rechazar la hipótesis específica 1 y se concluye que no existe impacto directo ni significativo entre la percepción de liderazgo autoritario coercitivo y el aprendizaje estudiantil.

\section{Tabla 8}

Prueba de correlación para contrastación de hipótesis específica 2

\begin{tabular}{|c|c|c|c|c|}
\hline & & & $\begin{array}{l}\text { Variable X } \\
\text { (dimensión 2) }\end{array}$ & Variable Y \\
\hline \multirow[t]{6}{*}{$\begin{array}{c}\text { Rho de } \\
\text { Spearman }\end{array}$} & Variable X: dimensión 2 & $\begin{array}{l}\text { Coeficiente de } \\
\text { correlación }\end{array}$ & 1,000 &, 257 \\
\hline & \multirow{5}{*}{$\begin{array}{l}\text { Liderazgo autoritario } \\
\text { benevolente } \\
\text { Variable Y: } \\
\text { Aprendizaje estudiantil }\end{array}$} & Sig. (bilateral) & . & 273 \\
\hline & & $\mathrm{N}^{\circ}$ & 20 & 20 \\
\hline & & $\begin{array}{l}\text { Coeficiente de } \\
\text { correlación }\end{array}$ &, 257 & 1,000 \\
\hline & & Sig. (bilateral) & ,273 & . \\
\hline & & $\mathrm{N}^{\circ}$ & 20 & 20 \\
\hline
\end{tabular}

Fuente: elaboración propia.

En la Tabla 8 se observa que existe un grado de correlación bajo entre la dimensión 2 de la variable X y la variable Y (Rho de Spearman: 0.257) a un nivel bilateral de significancia de 0.05 , es decir a un $95 \%$ de confianza. Debido a que el nivel crítico es inferior al nivel de significación establecido existen suficientes razones para rechazar la hipótesis específica 2 y se 
concluye que no existe impacto directo ni significativo entre la percepción de liderazgo autoritario benevolente y el aprendizaje estudiantil.

\section{Tabla 9}

Prueba de correlación para contrastación de hipótesis específica 3.

\begin{tabular}{|c|c|c|c|c|}
\hline & & & $\begin{array}{l}\text { Variable X } \\
\text { (dimensión 3) }\end{array}$ & Variable $\mathbf{Y}$ \\
\hline \multirow[t]{6}{*}{$\begin{array}{c}\text { Rho de } \\
\text { Spearman }\end{array}$} & \multirow[t]{3}{*}{$\begin{array}{l}\text { Variable X: dimensión } 3 \\
\text { Liderazgo consultivo }\end{array}$} & \multirow{3}{*}{$\begin{array}{l}\text { Coeficiente de } \\
\text { correlación } \\
\text { Sig. (bilateral) } \\
\mathrm{N}^{\circ}\end{array}$} & 1,000 & ,213 \\
\hline & & & &, 367 \\
\hline & & & 20 & 20 \\
\hline & \multirow[t]{3}{*}{$\begin{array}{l}\text { Variable Y: } \\
\text { Aprendizaje estudiantil }\end{array}$} & $\begin{array}{l}\text { Coeficiente de } \\
\text { correlación }\end{array}$ & ,213 & 1,000 \\
\hline & & Sig. (bilateral) &, 367 & $\cdot$ \\
\hline & & $\mathrm{N}^{\circ}$ & 20 & 20 \\
\hline
\end{tabular}

Fuente: elaboración propia.

En la Tabla 9 se observa que existe un grado de correlación bajo entre la dimensión 3 de la variable X y la variable Y (Rho de Spearman: 0.213 ) a un nivel bilateral de significancia de 0.05, es decir a 95\% de confianza. Debido a que el nivel crítico es inferior al nivel de significación establecido existen suficientes razones para rechazar la hipótesis específica 3 y se concluye que no existe impacto directo ni significativo entre la percepción de liderazgo consultivo y el aprendizaje estudiantil.

\section{Tabla 10}

Prueba de correlación para contrastación de hipótesis específica 4.

\begin{tabular}{lllll}
\hline & & & $\begin{array}{l}\text { Variable X } \\
\text { (dimensión 4) }\end{array}$ & Variable Y \\
\hline $\begin{array}{c}\text { Rho de } \\
\text { Spearman }\end{array}$ & Variable X: dimensión 4 & $\begin{array}{l}\text { Coeficiente de } \\
\text { correlación }\end{array}$ & 1,000 &, 305 \\
& Liderazgo participativo & $\begin{array}{l}\text { Sig. (bilateral) } \\
N^{\circ}\end{array}$ & 20 &, 192 \\
& Variable Y: & $\begin{array}{l}\text { Coeficiente de } \\
\text { correlación }\end{array}$ &, 305 & 20 \\
& Aprendizaje estudiantil & Sig. (bilateral) &, 192 & 1,000 \\
& & $\mathbf{N}^{\circ}$ & $\mathbf{2 0}$ & $\mathbf{2 0}$ \\
\hline
\end{tabular}

Fuente: elaboración propia.

En la Tabla 10 se observa que existe un grado de correlación bajo entre la dimensión 4 de la variable X y la variable Y (Rho de Spearman: 0.305) a un nivel bilateral de significancia de 
0.05, es decir a un $95 \%$ de confianza. Debido a que el nivel crítico es inferior al nivel de significación establecido existen suficientes razones para rechazar la hipótesis específica 4 y se concluye que no existe impacto directo ni significativo entre la percepción de liderazgo participativo y el aprendizaje estudiantil.

5) Conclusión: de acuerdo a la información muestral y con un nivel de significancia de $5 \%$, se rechaza la hipótesis general, es decir que no existe una relación directa y significativa entre el liderazgo directivo y el aprendizaje estudiantil en las instituciones educativas del Callao, 2019 (p-valor $=, 000<\alpha=0.05)$ y tiene una correlación negativa considerable (Rho de Spearman: 0,205).

\section{Discusión}

Estos hallazgos se asemejan a los encontrados en Perú por (Ricra, 2017) sin embargo, los resultados encontrados por (Martos, 2018) en La Libertad, (Casas, 2018) en Ate-Vitarte, (Campodinico, 2017) en Piura y (Chávez, 2017) en Cajamarca y a nivel internacional por (Alfonso, 2018) en Ibagué-Colombia, (Mina, 2018) en Cali-Colombia, (Prado y Paes, 2018) en Brasil, (Rios, Gonzales, Prierto y Moreno, 2017) en Bogotá, (Baeza, 2016) en Yucatán-México, (Reyes, 2016) en Medellín-Colombia y (Dou, Devos y Valcke) en China concluyeron que el liderazgo directivo transformacional impacta directa y significativamente con el aprendizaje estudiantil y otras variable tales como: gestión educativa, desempeño docente, satisfacción de los estudiantes, éxito escolar y la calidad educativa respectivamente. Del mismo modo, difiere completamente de los resultados obtenidos en el estudio internacional de (Aburizaizah, Kim y Fuller, 2019) el cual concluye que el liderazgo principal directivo positivamente influye en el aprendizaje estudiantil enmarcado en una descentralización de la gestión educativa en Arabia Saudita.

\section{Conclusiones}

Se concluye que no existe impacto directo ni significativo entre la percepción de los estilos de liderazgo directivo y el aprendizaje estudiantil en las instituciones educativas primarias del Callao, en base al coeficiente Rho de Spearman (0.205); por lo tanto, el aprendizaje estudiantil está asociado a factores múltiples como: el desempeño laboral docente, situación económica familiar, motivación estudiantil, etc. Asimismo, los estilos de liderazgo directivo propuestos por Likert (1968): autoritario coercitivo, en base al coeficiente Rho de Spearman (0.406); autoritario benevolente, en base al coeficiente Rho de Spearman (0.257); consultivo, en base al coeficiente Rho de Spearman (0.213) y participativo, en base al coeficiente Rho de Spearman (0.305); no inciden actualmente en el aprendizaje estudiantil porque no se enmarcan en una formación académica bajo un enfoque por competencias y de responsabilidad social.

\section{Recomendaciones}

Al ser una investigación innovadora en cuanto al tema "liderazgo directivo" y su impacto en el "aprendizaje estudiantil" en el nivel de educación básica regular primaria en la Provincia Constitucional del Callao, contribuye a ser punto de partida para futuras investigaciones que 
relacionen estas variables en otros tipos de centros educativos alternos como: Centros de Educación Básica Alternativa (CEBAS), Centros de Educación Básica Especial (CEBES), Programa de Intervención Temprana (PRITES), centros de estudios artísticos y de idiomas; desde perspectivas más complejas a nivel nacional e internacional.

No se debe difundir el estilo de liderazgo autoritario coercitivo en ninguno de los directores educativos porque no contribuye al desarrollo de competencias básicas, transversales y específicas en los docentes y estudiantes que están a su cargo.

No se debe de considerar el estilo de liderazgo autoritario benevolente en ninguno de los directores educativos porque interfiere con la veracidad de las relaciones interpersonales de los actores educativos y condiciona un clima organizacional bajo un proceso de recompensas y castigos explícitos.

Se debe de fomentar el estilo de transformacional con mayor énfasis en todos los directores de las instituciones educativas a nivel nacional puesto que, ha demostrado ser el que presenta resultados óptimos en las instituciones educativas; además, porque se adapta y comulga con la política democrática del país.

Se debe propiciar una nueva gestión educativa que fomente el aprendizaje estudiantil por competencias porque invita a la activa participación de todos los integrantes de la institución educativa, el mismo incluye a alumnos, ex alumnos, docentes, personal administrativo, padres de familia, etc.

\section{Referencias bibliográficas}

Aburizaizah, S., Kim, Y. y Fuller, B. (2019). Principal leadership and student achievement: decentralising school management in Saudi Arabia. Compare: A Journal of Comparative and International Education, 49(5), 795-816. Obtained from: https://doi.org/10.1080/03057925.2018.1462145

Alfonso, J. (2018). Liderazgo Directivo y Participación en la Gestión Escolar de la Institución Educativa Técnica Carlos Lleras Restrepo de Ibagué. Tesis maestría en educación, Universidad de Tolima. Obtenido de: http://repository.ut.edu.co/jspui/handle/001/2568

Araya, V., Alfaro, M., y Andonegui, M. (2007). Constructivismo: Orígenes y Perspectivas. Laurus, 13(24), 76-92. Obtenido de: https://www.redalyc.org/pdf/761/76111485004.pdf Baeza, O. (2016). Formas de Liderazgo a Nivel de Dirección en Escuelas Primarias.

Tesis maestría con mención en el campo de desarrollo curricular,

Universidad Pedagógica Nacional. Obtenido de: http://xplora.ajusco.upn.mx:8080/jspui/handle/123456789/1317

Campodonico, S. (2017). Estilos de Liderazgo de los Directores Académicos de una Institución de Educación Superior. Tesis maestría en educación con mención en teorías y prácticas 
educativas, Universidad de Piura. Obtenido de: https://pirhua.udep.edu.pe/handle/11042/2859

Casas, V. (2018). Liderazgo Pedagógico y Aprendizaje de los Estudiantes de la Institución Educativa Mixto Huaycán, Ate, 2018. Tesis maestría en Administración de la Educación, Universidad Cesar Vallejo. Obtenido de: http://repositorio.ucv.edu.pe/handle/UCV/22284?localeattribute $=$ en

Chávez, M. (2017). Relación entre el Liderazgo Directivo con el Desempeño Docente en las Instituciones Educativas Nuestra Señora de la Merced y Juan XXIII del Nivel SecundarioCajamarca, 2012. Tesis maestría en ciencias de la educación con mención en gestión de la educación, Universidad nacional de Cajamarca. Obtenido de: http://repositorio.unc.edu.pe/handle/UNC/2561

Chiavenato, i. (2004). Administración, teoría, proceso y práctica. (Tercera ed.). Madrid: McgrawHill.

Dou, D., Devos, G., y Valcke, M. (2016). The Relationships Between School Autonomy Gap, Principal Leadership, Tearchers' Job Satisfaction and Organizational Commitment. Sage Journals, 1. China. Obtained from: https://doi.org/10.1177/1741143216653975

Furguerle, J., y Vitorá, M. (2016). Liderazgo en los Directores de Educación Primaria. Telos. Revista de Estudios Interdisciplinarios en Ciencias Sociales. Universidad Privada Dr. Rafael Belloso Chacín, XVIII(2), 208-227. Obtenido de: https://www.redalyc.org/pdf/993/99345727004.pdf

Gardner, H. (1983). Inteligencias Múltiples. La teoría en la práctica. Barcelona: Paidos.

Garza, M., y Patiño, 1. (2000). Características del Liderazgo Social. (Segunda ed.). Bogotá: Magisterio.

Hernández, E., Santo, N., y Gónzalez, N. (2012). El Liderazgo. Psicología de los Grupos y Organizaciones. Murcia: Universidad Católica San Antonio de Murcia.

Likert, R. (1968). El Factor Humano en la Empresa. Su Dirección y Valoración. España: Deusto. Martos, R. (2018). Liderazgo Directivo y Desempeño Docente en la Institución Educativa de Educación Primaria $N^{\circ}$ 80386. Chepén- 2017. Tesis maestría en administración de la educación, Universidad Cesar Vallejo. Obtenido de: http://repositorio.ucv.edu.pe/handle/UCV/11837

Mina, C. (2018). Percepción del Estilo de Liderazgo del Director y Satisfacción de los Estudiantes del Bachillerato de la Corporación Educativa Adventista, Sede Cámbulos, de la ciudad de Cali, Colombia.Tesis maestría en educación, Universidad de Montemorelos. Obtenido de: http://dspace.biblioteca.um.edu.mx/xmlui/handle/20.500.11972/1009?show=full

Ministerio de Educación. (2009). Guía de Evaluación del Aprendizaje. Lima: MINEDU.

Palomino, F. (2012). El Desempeño Docente y el Aprendizaje de los Estudiantes de la Unidad Académica de Estudios Generales de la Universidad de San Martín de Porres. CybertesisUNMSM. Obtenido

de: http://cybertesis.unmsm.edu.pe/handle/cybertesis/1693 
Palomo, M. (2010). Liderazgo y Motivación de Equipos de Trabajo. (Sexta ed.). Madrid: Gráficas Dehon.

Popham, J. (2008). Classroom Assessment: what teachers need to know (Quinta ed.). Boston: Pearson Education.

Prado de Oliveira, A., y Paes de Carvalho, C. (2018). Public school management, leadership, and educational results in Brazil. Revista Brasileira de Educação, XXIII, 1. Obtido de: https://doi.org/10.1590/s1413-24782018230015

Reyes, N. (2012). Liderazgo Directivo y Desempeño Docente en el Nivel Secundario de una Institución Educativa de Ventanilla- Callao. Tesis maestría, Universidad San Ignacio de Loyola. Obtenido de: http://repositorio.usil.edu.pe/handle/123456789/1302

Reyes, N. (2016). Prácticas y Percepciones de Liderazgo del Directivo Docente. Tesis maestría en educación, Universidad de San Buenaventura Medellín. Obtenido de: http://bibliotecadigital.usbcali.edu.co/handle/10819/3368

Ricra, A. (2017). Liderazgo Directivo y Desempeño Docente en las Instituciones Educativas de Chorrillos - 2016. Tesis maestría en administración de la educación, Universidad Cesar Vallejo. Obtenido de: http://repositorio.ucv.edu.pe/handle/UCV/8292

Rios, M., Gonzales, i., Prieto, O., y Moreno, j. (2017). Liderazgo Directivo y su incidencia en el Éxito Escolar, como Factor de Calidad de la Institución Educativa en Cuatro Colegios Oficiales de Bogotá. Tesis maestría en Dirección y Gestión de Instituciones Educativas, Universidad de la Sabana. Obtenido de: https://intellectum.unisabana.edu.co/handle/10818/34630 Schunk, D. (2012). Teorías del Aprendizaje. Una Perspectiva Educativa. (Sexta ed.). Distrito Federal: Pearson Educación.

Unesco (2016). Organización de las Naciones Unidas para la Educación, la Ciencia y la Cultura. Obtenido de: http://www.unesco.org/new/es/media-services/singleview/news/unesco_school_leadership_is_crucial_to_improvement_in_stude/ 